\title{
THE IMPACT OF LIQUID BIO-GAS FERTILIZER ON FENNEL CROP PRODUCTIVITY AND OIL CONTENT UNDER DIFFERENT LEVELS OF IRRIGATION WATER
}

\author{
Mansour H. El-Bakhshwan ${ }^{1}$ and Elsayed H. Hassan ${ }^{2}$
}

\section{ABSTRACT:}

Two experiments were carried out at test and research station of Tractors and Farm machinery at Sabahia, Alexandria Governorate, Egypt during 2016/2017 season to study the effect of liquid Biogas fertilizer on vegetative growth, chemical composition and essential oil\% on fennel plants. The remaining mixture of the process of fermentation of organic and exogenous waste from the fermentation is called the name of the biogas fertilizer. This fertilizer can be dried and converted into organic liquid or dry fertilizer and used in the fertilization of various agricultural crops very efficiently and because of its reliable price and dispense with mineral fertilizers which are expensive and harmful to humans .The experimental design was split plot with three replicates. The main plots were conducted for two rates of liquid biogas fertilizer (15) and (25) $\mathrm{m}^{3} / \mathrm{fed}$ with Three levels of concentration of biogas fertilizer level 1 (10\%), Level 2 (20\%) and Level $3(30 \%)$ the subplots were occupied by three levels of irrigation (50\%, 75\% and 100\%)from the maximum irrigation rate of $4000 \mathrm{~m} 3 / \mathrm{fed}$. The main results could be summarized as follows (1) Application biogas liquid fertilizer level at $30 \%\left(25 \mathrm{~m}^{3} / \mathrm{Fed}\right)$ gave the highest mean values of all studied characters under the third level of water irrigation (50\%).Essential oil\% and majors compound \% for the Foeniculum vulgare tested in the study. The results also showed that the highest value for water use efficiency (WUE) was 0.56 for the treatment $\left(Z_{2} Q_{3} I_{3}\right)$ and the highest value for nitrogen fertilizer use efficiency (NUE) was 14.5 for the treatment $\left(Z_{1} Q_{1} I_{3}\right)$. However, all traits under study increased significantly due to biogas liquid fertilizer treatments over the control treatment

Keywords: fennel, liquid biogas fertilizer levels, irrigation levels, vegetative growth, Seed yield oil\%, chemical composition.

\footnotetext{
${ }^{1}$ Researcher, Agricultural Engineering Research Institute, Agriculture Research Centre, Egypt.

${ }^{2}$ Head researcher, Horticulture Research Institute Research Institute, Agriculture Research Centre, Egypt.
} 


\section{INTRODUCTION:}

Uennel (Feoeniculum vulgare, Mill) which belongs to the family
umbelliferae (Apiacecie) is a short lived herb, indigenous to
Europe and cultivated in India Chino and Egypt (Whichti and Bissel, 1994). It is an aromatic herb whose fruits contain essential of which is used for many purposes by human. The oil of fennel regulates the peristaltic functions of the gastrointestinal trace and relieves the spasms of intestines (Fathy et al., 2002). Extemally, the oil relieves muscular and rheumatic pains. Also, the seeds have a traditional reputation as an acid to weight loss and longevity. The major constituent of fennel oil is Anethole (Braun and Franz, 1999). Fertilization is one of the most important factors limiting the productivity of plant. The intensive uses of expensive mineral fertilizers in recent years result in environmental pollution problems. Chemical fertilizers at extremely high rates for a long period decreased the potential activity of microflora and the stability of soil organic matter, Pokorna (1984). Biofertilization is one of the most important factors used to produce products free from mineral contamination. On the other hand, the intensive uses of chemical fertilizer cause environmental pollution problems, utilized biofertilizers (phosphorus solubilizing microorganisms (Bacteria and Mycorrhizae) could supply plants with their needs of phosphorus during their growth. Also, bio-fertilizer play vital role for increasing the number of microorganisms and accelerate certain microbial process in the rhizosphare of inoculated soil which can change unavailable pormsto the available forms of nutrients for plants (Badawi, 2000, Kandell et al., 2001 and Massoud, 2007). The remaining mixture of the process of fermentation of organic and exogenous waste from the fermentation is called the name of the liquid biogas fertilizer. This fertilizer can be dried and converted into organic liquid or dry fertilizer and used in the fertilization of various agricultural crops very efficiently and because of its reliable price and dispense with mineral fertilizers which are expensive and harmful to humans. Therefore, the liquid manure was selected in this research and its effect on the fennel plant in the different stages of cultivation and on productivity and on the percentage of extracted oil.Many rehearses reported the use of biogas residues as soil fertilizer 
with success (Maunksela et al., 2012Diacono \& Montemurro., 2011; Haraldsen et $\boldsymbol{a l}$., 2011). The residues generated from biogas processes have a higher concentration of NH4 compared with conventional animal manure and compost hence their potential fertilizer value is also higher (Zhong et al ., 2010).In addition most elemental nutrients such as $\mathrm{P} K$ $\mathrm{Mg}$ and a number of other essential trace elements from the raw material fed to the biogas process ram in the biogas residue ( Abubaker., 2012). The organic compounds not broken down during the biogas process increase the carbon content of farmland and improve its biological activity as they break in the soil. The studied factors included irrigation treatment (at three levels of irrigation to $50 \%, 75 \%$ and $100 \%$ of reference evapotranspiration Therefore, the objective of this study were: (1) Evaluation the effect of biogas fertilizer and water irrigation levels and their interaction on the fennel growth, yield, essential oil chemical composition. (2) Water use efficiency (WUE). (3) Nitrogen fertilizer use efficiency (NUE).

\section{MATERIALS AND METHODS:}

\subsection{Experimental site}

The experiments were carried out at the Test and Research station of Tractors and Farm machinery (Sabhia), during 2016/2017 season. The applied experimental design was split plot with three replicates. The main plots were conducted for two rate of biogas fertilizer (liquid) by the three levels and the subplots were occupied by the three levels of water irrigation treatments $(50 \%, 75 \%$ and $100 \%)$ Added recommended dose of NPK fertilizer to control treatment.

\subsection{Levels of irrigation}

Three levels of irrigation and quantities of water applied According to the Desert Research Center (2010).

1 - $4000 \mathrm{~m}^{3} /$ Fadden represents the maximum irrigated water rate for

Fennel plant (i.e $100 \%$ of water requirements (WR).

2- $3000 \mathrm{~m}^{3} /$ Fadden represents the maximum irrigated water rate for

Fennel plant (i.e $75 \%$ of water requirements (WR).

3- $2000 \mathrm{~m}^{3} /$ Fadden represents the maximum irrigated water rate for Fennel plant (i.e $50 \%$ of water requirements (WR). 


\subsection{Soil description}

Soil samples were collected to determine some physical and chemical characteristics of the investigated soil were determined according to Page et al. (1982) is shown in Table (1).

\section{The chemical analysis of water}

Analyzed in Saline and Alkaline research Lab Alexandria

The chemical analysis of water was $0.9,0.25,0.00,0.3,0.8,15$ and 8 (meq/L) for $\mathrm{Ca}, \mathrm{Mg}, \mathrm{CO} 3, \mathrm{HCO} 3, \mathrm{CL}, \mathrm{Na}$ and $\mathrm{K}$, respectively.

Table (1) some physical and chemical properties of the tested soil.

\begin{tabular}{|l|c|}
\hline \multicolumn{1}{|c|}{ Properties } & The values \\
\hline \multicolumn{2}{|c|}{ Chemical properties } \\
\hline PH (1:2.5) soil : water & 7.95 \\
\hline EC,d S m-1 (1:2.5) & 2.60 \\
\hline Total nitrogen, \% (T.N) & 0.10 \\
\hline Available nitrogen ,ppm (Av.N) & 24.24 \\
\hline \multicolumn{2}{|c|}{ Physical properties } \\
\hline Total porosity, (T.P) \% & 51.25 \\
\hline Hydraulic Conductively (HC) (cm/h) & 01.75 \\
\hline Available water, (Av.W) \% & 17.95 \\
\hline Texture class & Sand clay loam \\
\hline Particle size distribution, \% & 44.20 \\
1- Sand & 26.10 \\
2- Silt & 29.76 \\
3- Clay & \\
\hline
\end{tabular}

\subsection{Planting treatments and experimental design}

The experiment was performed in split split- plot design in a randomized complete block design (RCBD) with three replicates.

Treatments consisted of two rates of biogas fertilizer 15 and 25 cubic meters / fed, the subplots were occupied by Three concentrations of liquid fertilizer (10, 20 and 30\%) and water regimes (control, i.e. three rates of irrigation: ( $50 \%, 75 \%$ and 100\%) represent $(2000,3000$, and 4000 $\mathrm{m}^{3} /$ fed). Fennel plant was sown in the soil in rows of $10 \mathrm{~m}$ length and 1.0 $\mathrm{m}$ apart. The spacing between rows were $25 \mathrm{~cm}$, the seeds were sown in holes with $25 \mathrm{~cm}$ within a row, beside $3-4$ seeds were sown in every hole. Thinning to one plant per hole was performed 40 days after sowing. The plot area was $(3 \times 3 \mathrm{~m})$ and contained 3 rows. There were 12 plants in every row 36 plant in each plot. Seeds of fennel were obtained from Medicinal 
and Aromatic Plants Department Agricultural Research center, Egypt, seeds were sown on Nov 12th during the successive season 2016/2017. The same design was followed with flooding irrigation system. The plants were irrigated by flooding irrigation up to the field capacity ( $4000 \mathrm{~m} 3 / \mathrm{fed})$. The plants were harvested on $25^{\text {th }}$ April during the season and the following data were recorded: plant height $(\mathrm{cm})$, number of main branches per plant, number of umbels per plant, diameter of umber per plant, weight of fruits per fed $(\mathrm{kg})$.

\subsection{Biogas liquid manure application rates}

The liquid biogas manure brought from the biogas project located at Test and Research station of Tractors and Farm machinery, Sabahia, Alexandria Governorate, Egypt. The components of the liquid biogas manure were analyzed in the laboratories of the services unit of soil analysis, Soil Department, Faculty of Agriculture, Alexandria University, Egypt.

Table (2) shows characteristics of the investigated liquid biogas manure.

\begin{tabular}{|c|c|c|c|c|c|c|c|c|c|c|c|}
\hline Sample & $\mathrm{N}$ & $\mathrm{P}$ & $\mathrm{K}$ & $\mathrm{Fe}$ & $\mathrm{Zn}$ & $\mathrm{Cu}$ & $\mathrm{Cd}$ & $\mathrm{Ni}$ & $\mathrm{Cr}$ & $\mathrm{Pb}$ \\
\cline { 2 - 11 } & \multicolumn{3}{|c|}{$\%$} & \multicolumn{7}{c|}{$\mathrm{ug} / \mathrm{g}, \mathrm{ug} / \mathrm{ml}$} \\
\hline $\begin{array}{c}\text { Fresh } \\
\text { Dung }\end{array}$ & 1.27 & 0.48 & 1.40 & 1952.00 & 60.00 & 20.00 & 34.00 & 66.0 & 0.00 & 22.00 \\
\hline $\begin{array}{c}\text { Liquid } \\
\text { fertilizer }\end{array}$ & 1.13 & 0.52 & 2.10 & 124.00 & 7.60 & 2.40 & 3.40 & 8.00 & 0.00 & 5.40 \\
\hline
\end{tabular}

\section{Determination of oil percentage:}

The essential oil percentage in the fennel seeds was determined according to British Pharmacopoeia (1963).Satisfactory results were obtained by distilling $100 \mathrm{~g}$ seeds for $1.5-2.0$ hours.

\section{Chemical composition of essential oil:}

Chemical composition of the extracted essential oil of fennel was identified using a Thermo scientific GC/MS version (5) 2009 system with TG-5MS column (30mX0.32mmID). Helium was used as a carrier gas at a flow rate of $1 \mathrm{ml} / \mathrm{min}$. Five $\mu \mathrm{l}$ essential oil was diluted to $1 \mathrm{ml}$ with dichloromethane, then $2 \mu \mathrm{l}$ was injected on splitless mode for $1 \mathrm{~min}$. followed by a split flow with ratio 1:10. GC oven temperature was held at $45^{\circ} \mathrm{C}$ for $2 \mathrm{~min}$ then was programmed from $45^{\circ} \mathrm{c}$ to $165^{\circ} \mathrm{C}$ at $4^{\circ} \mathrm{C} / \mathrm{min}$; from $165^{\circ} \mathrm{C}$ to $280^{\circ} \mathrm{C}$ at $15^{\circ} \mathrm{C} / \mathrm{min}$. after which was kept constant at $280^{\circ} \mathrm{C}$ for $10 \mathrm{~min}$. Both the interface and injection temperatures were adjusted at 
$250^{\circ} \mathrm{C}$. The ionization voltage was $70 \mathrm{eV}$ with a mass range between $40-$ 800mandz. The essential oil components were identified by mass fragmentation patterns, which were compared with NIST mass spectral database (version 2) and their relative percentages were calculated based on GC peak areas

\section{Water use efficiency (WUE):}

Water use efficiency is the measure of a cropping system's capacity to convert water into plant biomass or grains. Water use efficiency (WUE) was determined according to Michael (1978) by using the following equation,

Water use efficiency $=$ Crop yield,$(\mathrm{kg} / \mathrm{Fed}) /$ Water applied, $\left(\mathrm{m}^{3 /} \mathrm{Fed}\right)$

\section{Nitrogen fertilizer use efficiency (NUE):}

Table (3) shows: quantities of nitrogen units per treatment Fertilizer use efficiency was calculated for each treatment according to the following relation by Jensen (1983):

NUE $=$ Total Fresh Yield $(\mathrm{kg}) /$ Total Applied Nitrogen Fertilizer $(\mathrm{kg}-\mathrm{N})$

Statistical Analysis:

All the data obtained were statistical subjected to analysis of variance as described by Gomez and Gomez (1984); using L.S.D. at 0.05.

Table (3) shows: quantities of nitrogen units per treatment Fertilizer

\begin{tabular}{|c|c|c|}
\hline \multicolumn{2}{|c|}{ Treatments } & unit's of nitrogen \\
\hline \multirow{3}{*}{$\mathrm{Z}_{1}$} & $\mathrm{Q}_{1}$ & 169.5 \\
\cline { 2 - 3 } & $\mathrm{Q}_{2}$ & 113 \\
\cline { 2 - 3 } & $\mathrm{Q}_{3}$ & 56.5 \\
\hline \multirow{3}{*}{$\mathrm{Z}_{2}$} & $\mathrm{Q}_{1}$ & 282.5 \\
\cline { 2 - 3 } & $\mathrm{Q}_{2}$ & 188.33 \\
\cline { 2 - 3 } & $\mathrm{Q}_{3}$ & 94 \\
\hline
\end{tabular}

\section{RESULTS AND DISCUSSION}

\section{Plant height and Seed yield:}

Data presented in Table (4) showed that with increasing liquid fertilizer levels significantly, increased the plant height with significant difference for the most liquid fertilizer levels under all irrigation level compare with control treatment However, the tallest plants were observed for the application $\left(\mathrm{Z}_{2}\right.$ at $\mathrm{Q}_{3}$ in $\left.\mathrm{I}_{3}=145.01 \mathrm{~cm} / \mathrm{plant}\right)$, As well as the highest productivity was for the application $\left(\mathrm{Z}_{2}\right.$ at $\mathrm{Q}_{3}$ in $\left.\mathrm{I}_{3}=1120 \mathrm{~kg} / \mathrm{fed}\right)$. 
While the shortest plants and Less productive were recorded by applied the control treatment $=110 \mathrm{~cm} /$ plant, $670 \mathrm{~kg} / \mathrm{fed}$, respectively.

Table (4): Effect of Liquid Bio-Gas Fertilizer on Fennel crop productivity under different levels of irrigation water

\begin{tabular}{|c|c|c|c|c|c|c|}
\hline \multicolumn{3}{|c|}{ Treatments } & $\begin{array}{c}\text { Plant } \\
\text { Height } \\
\text { (cm) }\end{array}$ & $\begin{array}{l}\text { Branches } \\
\text { Number }\end{array}$ & $\begin{array}{l}\text { Inflorescences } \\
\text { Number/Plant }\end{array}$ & $\begin{array}{l}\text { Seed Yield } \\
\text { /Fed (kg) }\end{array}$ \\
\hline \multicolumn{3}{|c|}{ Control } & 110.00 & 4.33 & 18.88 & 670 \\
\hline \multirow{9}{*}{$\mathrm{Z}_{1}$} & \multirow{3}{*}{$\mathrm{Q}_{1}$} & $\mathrm{I}_{1}$ & 121.31 & 4.21 & 29.76 & 746 \\
\hline & & $\mathrm{I}_{2}$ & 122.23 & 4.38 & 31.15 & 785 \\
\hline & & $\mathrm{I}_{3}$ & 125.96 & 4.96 & 32.13 & 821 \\
\hline & \multirow{3}{*}{$\mathrm{Q}_{2}$} & $\mathrm{I}_{1}$ & 128.17 & 4.17 & 35.23 & 776 \\
\hline & & $\mathrm{I}_{2}$ & 128.12 & 4.83 & 36.15 & 811 \\
\hline & & $\mathrm{I}_{3}$ & 129.11 & 5.17 & 33.86 & 766 \\
\hline & \multirow{3}{*}{$\mathrm{Q}_{3}$} & $\mathrm{I}_{1}$ & 128.98 & 4.98 & 33.21 & 776 \\
\hline & & $\mathrm{I}_{2}$ & 130.29 & 5.33 & 33.17 & 893 \\
\hline & & $\mathrm{I}_{3}$ & 131.26 & 5.81 & 34.83 & 911 \\
\hline \multirow{9}{*}{$\mathrm{Z}_{2}$} & \multirow{3}{*}{$\mathrm{Q}_{1}$} & $\mathrm{I}_{1}$ & 132.16 & 5.36 & 35.45 & 793 \\
\hline & & $\mathrm{I}_{2}$ & 132.36 & 5.78 & 35.83 & 825 \\
\hline & & $\mathrm{I}_{3}$ & 135.86 & 6.1 & 36.13 & 827 \\
\hline & \multirow{3}{*}{$\mathrm{Q}_{2}$} & $\mathrm{I}_{1}$ & 133.50 & 5.9 & 35.22 & 963 \\
\hline & & $\mathrm{I}_{2}$ & 137.09 & 6.2 & 36.99 & 980 \\
\hline & & $\mathrm{I}_{3}$ & 138.20 & 6.9 & 43.93 & 1033 \\
\hline & \multirow{3}{*}{$\mathrm{Q}_{3}$} & $\mathrm{I}_{1}$ & 137.30 & 6.8 & 43.07 & 997 \\
\hline & & $\mathrm{I}_{2}$ & 141.60 & 7.5 & 47.17 & 1080 \\
\hline & & $\mathrm{I}_{3}$ & 145.01 & 8.0 & 48.34 & 1120 \\
\hline \multicolumn{3}{|c|}{ L.S.D 0.05} & 4.05 & 1.21 & 2.20 & 10.09 \\
\hline
\end{tabular}

Where in:

Rate of liquid fertilizer:

$\mathrm{Z}_{1}=15 \mathrm{~m}^{3} / \mathrm{fed} \quad \mathrm{Z}_{2}=25 \mathrm{~m}^{3} / \mathrm{fed}$

Concentrations of liquid fertilizer

$\mathrm{Q}_{1}=10 \% \quad \mathrm{Q}_{2}=20 \% \quad \mathrm{Q}_{3}=30 \%$

Water rate of transpiration evaporation:

$\mathrm{I}_{1}=100 \% \quad \mathrm{I}_{2}=75 \% \quad \mathrm{I}_{3}=50 \%$

Branches Number, Inflorescences Number/Plant and seed yield:

Data presented in Table (4) showed that, also with increasing liquid fertilizer levels significantly, increased the Branches Number, Inflorescences Number/Plant and seed yield with significant difference 
for the most liquid fertilizer levels under all irrigation level compare with control treatment. The promoting effect of liquid fertilizer levels on plant height could be attributed to the fundamental role of fertilizer factors as a structural [art of many compounds as nucleic acid and phospholipids (Weier,et. Al. 1993). This may be due to the increase of growth root net - zone. Also, the solubilization of mineral nutrients synthesis of vitamin, phospholipids, nuclic acid and gibberellins (Sadej, W. \& Przekwas, K. ,2008). Data presented in Table (5) showed that with increasing liquid fertilizer levels significantly, increased essential oil percentage in seed fennel.

\section{Essential oil and major's components \%:}

Table (5): Effect of Liquid Bio-Gas Fertilizer on Fennel oil and majors components \% under different levels of irrigation water

\begin{tabular}{|c|c|c|c|c|c|c|}
\hline \multicolumn{3}{|c|}{ Treatments } & \multirow{2}{*}{$\begin{array}{c}\text { Essential } \\
\text { Oil \% }\end{array}$} & \multirow{2}{*}{$\begin{array}{c}\begin{array}{c}\text { Anethol } \\
\%\end{array} \\
42.52\end{array}$} & \multirow{2}{*}{$\begin{array}{c}\text { Fenchone } \\
\%\end{array}$} & \multirow{2}{*}{$\begin{array}{c}\begin{array}{c}\text { Methyl } \\
\text { chavicol } \\
\%\end{array} \\
4.19\end{array}$} \\
\hline \multicolumn{3}{|c|}{ Control } & & & & \\
\hline \multirow{9}{*}{$\mathrm{Z}_{1}$} & \multirow{3}{*}{$\mathrm{Q}_{1}$} & $\mathrm{I}_{1}$ & 1.59 & 52.51 & 8.05 & 3.56 \\
\hline & & $\mathrm{I}_{2}$ & 1.62 & 57.45 & 8.29 & 3.39 \\
\hline & & $\mathrm{I}_{3}$ & 1.59 & 58.01 & 9.12 & 3.35 \\
\hline & \multirow{3}{*}{$\mathrm{Q}_{2}$} & $\mathrm{I}_{1}$ & 1.58 & 58.31 & 9.04 & 3.31 \\
\hline & & $\mathrm{I}_{2}$ & 1.62 & 58.24 & 9.35 & 3.25 \\
\hline & & $\mathrm{I}_{3}$ & 1.61 & 60.01 & 9.55 & 3.25 \\
\hline & \multirow{3}{*}{$\mathrm{Q}_{3}$} & $\mathrm{I}_{1}$ & 1.68 & 59.14 & 9.64 & 3.21 \\
\hline & & $\mathrm{I}_{2}$ & 1.69 & 60.08 & 9.64 & 3.18 \\
\hline & & $\mathrm{I}_{3}$ & 1.69 & 60.55 & 9.67 & 3.15 \\
\hline \multirow{9}{*}{ rZ } & \multirow{3}{*}{$\mathrm{Q}_{1}$} & $\mathrm{I}_{1}$ & 1.68 & 61.81 & 9.70 & 3.06 \\
\hline & & $\mathrm{I}_{2}$ & 1.76 & 61.05 & 9.70 & 3.03 \\
\hline & & $\mathrm{I}_{3}$ & 1.62 & 63.51 & 9.82 & 3.02 \\
\hline & \multirow{3}{*}{$\mathrm{Q}_{2}$} & $\mathrm{I}_{1}$ & 2.00 & 65.12 & 9.79 & 3.04 \\
\hline & & $\mathrm{I}_{2}$ & 2.00 & 66.23 & 9.81 & 3.05 \\
\hline & & $\mathrm{I}_{3}$ & 2.01 & 67.61 & 9.84 & 3.05 \\
\hline & \multirow{3}{*}{$\mathrm{Q}_{3}$} & $\mathrm{I}_{1}$ & 2.02 & 64.23 & 9.83 & 3.04 \\
\hline & & $\mathrm{I}_{2}$ & 2.06 & 68.08 & 9.88 & 3.07 \\
\hline & & $\mathrm{I}_{3}$ & 2.11 & 68.81 & 9.93 & 3.09 \\
\hline \multicolumn{3}{|c|}{ L.S.D 0.05} & 0.03 & 1.07 & 0.28 & 0.02 \\
\hline
\end{tabular}

The mean of essential oil percentage per plant increased due to increasing up to Fertilizer rate of $25 \mathrm{~m}^{3}$ / fed and the contrition of liquid fertilizer up to $\mathrm{Q}_{3}=30 \%$ under third irrigation level $=50 \%$, while the lowest one was 
recorded by control treatment, respectively. These may be due to the increase availability of fertilizer factors up take by plants at considerable rate to build up more metabolites necessary for including the volatile oil synthesis.

\section{Effect of liquid Biogas fertilizer and different levels of irrigation water on soil after harvesting:}

Effect of liquid Biogas fertilizer and different levels of irrigation water on total nitrogen (T.N) and available nitrogen (Av.N) and some soil sample properties collected from rhizosphere area of grown fennel plant after harvesting showed in table (6).

The obtained data showed, the effect of two rates of liquid biogas fertilizer (15) and (25) $\mathrm{m} 3$ / fed, three levels of concentration of biogas fertilizer [ level 1 (10\%), Level $2(20 \%)$ and Level $3(30 \%)$ ] and three levels of irrigation $(\mathbf{5 0 \%}, \mathbf{7 5 \%}$ and $\mathbf{1 0 0 \%})$ ) respectively on soil, which caused a pronounced amelioration effect on available nitrogen (Av.N), total nitrogen (T.N) and some soil properties as, soil PH, available water (Av.W) and as a shown in table (6). Data illustrated in table (6) showed a progressive significant increase in the all treatments compared the control treatment. The greatest mean value of available nitrogen (Av.N) was $132.25 \mathrm{ppm}$ with $\mathrm{Z}_{2} \mathrm{Q}_{3} \mathrm{I}_{3}$. The positive response of applied organic manure and mineral fertilizer as well as water requirements was displayed by many authors such as Mohamed (2006) and beside its beneficial effect on some soil chemical these finding were confirmed by the results founded by Saker et al (1992).

Point of view, the combined treatment of $\mathrm{Z}_{2} \mathrm{Q}_{2} \mathrm{I}_{1}$ which is considered the superiority over than the other treatments was great enough to reach the level of significance under the prevailing conditions of current experiment. The greatest mean value for $\mathrm{Z}_{2} \mathrm{Q}_{3} \mathrm{I}_{3}$ was $26.48 \%$ for values (Av. W) (Ewees et al 2008). Table (5) represent the mean values of total nitrogen (T.N) and available nitrogen ( Av.N) in all treatments in soil. Data indicated a progressive significant increase in all treatments where the greatest mean value of total nitrogen (T.N) and available nitrogen ( Av.N) were $2.15 \%$ and 132.25 ppm respectively with $\mathrm{Z}_{2} \mathrm{Q}_{3} \mathrm{I}_{3}$.

The lowest mean values were with control,(Abou EL-Maged et al 2006). 


\section{Water use efficiency (WUE):}

Table (7) indicates the average values of water use efficiency obtained in all treatments. The higher water use efficiency were 0.56 and $0.52 \mathrm{~kg} / \mathrm{m}^{3}$ gained from the treatment $\left[\mathrm{Z}_{2} \mathrm{Q}_{3} \mathrm{I}_{3}\right]$, and the treatment $\left[\mathrm{Z}_{2} \mathrm{Q}_{2} \mathrm{I}_{3}\right]$ respectively compared with the control treatment which was $0.17 \mathrm{~kg} / \mathrm{m}^{3}$.

\section{Nitrogen fertilizer use efficiency (NUE):}

Table (8) indicates the average values of nitrogen use efficiency obtained in all treatments. The higher nitrogen fertilizer use efficiency was 14.5 and 13.8 gained from the treatment $\left[\mathrm{Z}_{1} \mathrm{Q}_{1} \mathrm{I}_{3}\right]$, and the treatment $\left[\mathrm{Z}_{1} \mathrm{Q}_{1} \mathrm{I}_{2}\right]$ respectively compared with the rest of the treatments. While the lowest value of the treatment $\left[\mathrm{Z}_{2} \mathrm{Q}_{3} \mathrm{I}_{1}\right]$

Table (6): Effect of liquid Biogas fertilizer and different levels of irrigation water on soil after harvesting:

\begin{tabular}{|c|c|c|c|c|c|c|}
\hline \multicolumn{3}{|c|}{ TREATMENTS } & \multirow{2}{*}{$\begin{array}{c}\text { PH } \\
1: 2.5\end{array}$} & \multirow{2}{*}{$\begin{array}{c}\text { Av.W } \\
\%\end{array}$} & \multirow[t]{2}{*}{ T.N \% } & \multirow{2}{*}{$\begin{array}{l}\text { Av.N } \\
\text { ppm }\end{array}$} \\
\hline $\mathrm{m}^{3} / \mathrm{fed}$ & Depth $(\mathrm{cm})$ & Times & & & & \\
\hline \multicolumn{3}{|c|}{ CONTROL } & 7.52 & 14.70 & 0.105 & 72.25 \\
\hline \multirow{9}{*}{$\mathrm{Z}_{1}$} & \multirow[t]{3}{*}{$\mathrm{Q}_{1}$} & $\mathrm{I}_{1}$ & 7.73 & 20.21 & 1.59 & 109.12 \\
\hline & & $\mathrm{I}_{2}$ & 7.81 & 21.25 & 1.62 & 111.21 \\
\hline & & $\mathrm{I}_{3}$ & 7.69 & 22.92 & 1.65 & 118.35 \\
\hline & \multirow[t]{3}{*}{$\mathrm{Q}_{2}$} & $\mathrm{I}_{1}$ & 7.61 & 21.02 & 1.69 & 109.16 \\
\hline & & $\mathrm{I}_{2}$ & 7.96 & 21.89 & 1.73 & 112.52 \\
\hline & & $\mathrm{I}_{3}$ & 7.75 & 24.55 & 1.95 & 126.85 \\
\hline & \multirow[t]{3}{*}{$\mathrm{Q}_{3}$} & $\mathrm{I}_{1}$ & 7.71 & 22.12 & 1.60 & 112.21 \\
\hline & & $\mathrm{I}_{2}$ & 7.75 & 22.82 & 1.68 & 118.95 \\
\hline & & $\mathrm{I}_{3}$ & 7.81 & 23.03 & 1.73 & 121.26 \\
\hline \multirow{9}{*}{$\mathrm{Z}_{2}$} & \multirow[t]{3}{*}{$\mathrm{Q}_{1}$} & $\mathrm{I}_{1}$ & 7.33 & 23.86 & 1.97 & 116.12 \\
\hline & & $\mathrm{I}_{2}$ & 7.51 & 24.56 & 1.98 & 117.21 \\
\hline & & $\mathrm{I}_{3}$ & 7.48 & 24.78 & 2.12 & 122.35 \\
\hline & \multirow[t]{3}{*}{$\mathrm{Q}_{2}$} & $\mathrm{I}_{1}$ & 7.55 & 25.24 & 2.04 & 121.01 \\
\hline & & $\mathrm{I}_{2}$ & 7.71 & 25.43 & 1.88 & 121.23 \\
\hline & & $\mathrm{I}_{3}$ & 7.62 & 26.11 & 1.98 & 126.88 \\
\hline & \multirow[t]{3}{*}{$\mathrm{Q}_{3}$} & $\mathrm{I}_{1}$ & 7.59 & 25.66 & 1.55 & 122.56 \\
\hline & & $\mathrm{I}_{2}$ & 7.66 & 26.09 & 2.01 & 127.33 \\
\hline & & $\mathrm{I}_{3}$ & 7.89 & 26.48 & 2.15 & 132.25 \\
\hline \multicolumn{3}{|c|}{ L.S.D at 0.05} & 1.81 & 0.21 & 0.19 & 8.92 \\
\hline
\end{tabular}


Table (7): Average values of water use efficiency.

\begin{tabular}{|c|c|c|c|c|c|}
\hline \multicolumn{3}{|c|}{ Treatment } & $\begin{array}{l}\text { Yield } \\
\mathrm{kg} / \text { Fed }\end{array}$ & $\begin{array}{c}\text { Water irrigation, } \\
\text { m3/Fed }\end{array}$ & $\begin{array}{l}\text { W U E } \\
\mathrm{kg} / \mathrm{m} 3\end{array}$ \\
\hline \multicolumn{3}{|c|}{ Control } & 670 & 4000 & 0.17 \\
\hline \multirow{9}{*}{$Z_{1}$} & \multirow{3}{*}{$\mathrm{Q}_{1}$} & $\mathrm{I}_{1}$ & 746 & 4000 & 0.19 \\
\hline & & $\mathrm{I}_{2}$ & 785 & 3000 & 0.26 \\
\hline & & $\mathrm{I}_{3}$ & 821 & 2000 & 0.41 \\
\hline & \multirow{3}{*}{$\mathrm{Q}_{2}$} & $\mathrm{I}_{1}$ & 776 & 4000 & 0.19 \\
\hline & & $\mathrm{I}_{2}$ & 811 & 3000 & 0.27 \\
\hline & & $\mathrm{I}_{3}$ & 766 & 2000 & 0.39 \\
\hline & \multirow{3}{*}{$\mathrm{Q}_{3}$} & $I_{1}$ & 776 & 4000 & 0.19 \\
\hline & & $\mathrm{I}_{2}$ & 893 & 3000 & 0.30 \\
\hline & & $\mathrm{I}_{3}$ & 911 & 2000 & 0.46 \\
\hline \multirow{9}{*}{$\mathrm{Z}_{2}$} & \multirow{3}{*}{$\mathrm{Q}_{1}$} & $I_{1}$ & 793 & 4000 & 0.20 \\
\hline & & $\mathrm{I}_{2}$ & 825 & 3000 & 0.28 \\
\hline & & $\mathrm{I}_{3}$ & 827 & 2000 & 0.41 \\
\hline & \multirow{3}{*}{$\mathrm{Q}_{2}$} & $I_{1}$ & 963 & 4000 & 0.24 \\
\hline & & $\mathrm{I}_{2}$ & 980 & 3000 & 0.33 \\
\hline & & $\mathrm{I}_{3}$ & 1033 & 2000 & 0.52 \\
\hline & \multirow{3}{*}{$\mathrm{Q}_{3}$} & $\mathrm{I}_{1}$ & 997 & 4000 & 0.25 \\
\hline & & $\mathrm{I}_{2}$ & 1080 & 3000 & 0.36 \\
\hline & & $\mathrm{I}_{3}$ & 1120 & 2000 & 0.56 \\
\hline
\end{tabular}

Table (8): Average values of nitrogen use efficiency.

\begin{tabular}{|c|c|c|c|c|c|}
\hline \multicolumn{3}{|c|}{ Treatment } & Yield kg/Fed & $\begin{array}{c}\text { Nitrogen } \\
\text { Fertilizer }(\mathrm{kg}-\mathrm{N}\end{array}$ & $\begin{array}{c}\text { N U E } \\
\mathrm{kg} / \mathrm{N} \text {. unit }\end{array}$ \\
\hline \multirow{9}{*}{$\mathrm{Z}_{1}$} & \multirow{3}{*}{$\mathrm{Q}_{1}$} & $\mathrm{I}_{1}$ & 746 & 56.5 & 13.2 \\
\hline & & $\mathrm{I}_{2}$ & 785 & 56.5 & 13.8 \\
\hline & & $\mathrm{I}_{3}$ & 821 & 56.5 & 14.5 \\
\hline & \multirow{3}{*}{$\mathrm{Q}_{2}$} & $\mathrm{I}_{1}$ & 776 & 113 & 6.87 \\
\hline & & $\mathrm{I}_{2}$ & 811 & 113 & 7.18 \\
\hline & & $\mathrm{I}_{3}$ & 766 & 113 & 6.78 \\
\hline & \multirow{3}{*}{$\mathrm{Q}_{3}$} & $\mathrm{I}_{1}$ & 776 & 169.5 & 4.58 \\
\hline & & $\mathrm{I}_{2}$ & 893 & 169.5 & 5.27 \\
\hline & & $\mathrm{I}_{3}$ & 911 & 169.5 & 5.38 \\
\hline \multirow{9}{*}{$\mathrm{Z}_{2}$} & \multirow{3}{*}{$\mathrm{Q}_{1}$} & $I_{1}$ & 793 & 94 & 8.44 \\
\hline & & $\mathrm{I}_{2}$ & 825 & 94 & 8.78 \\
\hline & & $\mathrm{I}_{3}$ & 827 & 94 & 8.80 \\
\hline & \multirow{3}{*}{$\mathrm{Q}_{2}$} & $\mathrm{I}_{1}$ & 963 & 188.33 & 5.11 \\
\hline & & $\mathrm{I}_{2}$ & 980 & 188.33 & 5.20 \\
\hline & & $\mathrm{I}_{3}$ & 1033 & 188.33 & 5.49 \\
\hline & \multirow{3}{*}{$\mathrm{Q}_{3}$} & $\mathrm{I}_{1}$ & 997 & 282.5 & 3.53 \\
\hline & & $\mathrm{I}_{2}$ & 1080 & 282.5 & 3.82 \\
\hline & & $\mathrm{I}_{3}$ & 1120 & 282.5 & 3.97 \\
\hline
\end{tabular}




\section{CONCULATIONS}

\section{The results indicated the following:}

The treatment $\left(\mathrm{Z}_{2} \mathrm{Q}_{3} \mathrm{I}_{3}\right)$ was the greatest significant of total nitrogen concentrations (T.N), available nitrogen (Av.N), and (Av.W) of all other treatments as well as control treatment.

Addition of $\mathrm{Z}_{2}$ Fertilizer rate $\left(25 \mathrm{~m}^{3} / \mathrm{fed}\right), \mathrm{Q}_{3}$ concentration $\left.(30 \%)\right)$ and $\mathrm{I}_{3}(50 \% \mathrm{WR})$ resulted in high significant values of plant height $(\mathrm{cm})$, number of main branches per plant, number of umbels per plant, and weight of fruits per fed $(\mathrm{kg})$

\section{REFERENCES:}

Abou El-maged. M.M., A.M.,El bassiony. and Z. F.Fawzy. (2006) Effect of organic manure with or without chemical fertilizers on growth yidd and quilety of some varieties of broccoli plants. $j$. Appll. Sci. res. 2 (16): 791-798.

Abubaker, J. (2012). Effect of fertilization with biogas residues on crop yield, soil microbiology and greenhouse gas emission. Ph D thesis Swedish University of Agricultural Sciences, Uppsala, Sweden.

Badawi, A. M. A. (2000). Effect of some agricultural treatments on growth and active ingredients in roselle plants in North Sinai. Ph. D. Thesis, Fac. Agric. Zagzig Univ., Egypt.

Braun, M. and G. Franz (1999). Quality criteria of bitter fennel oil in the German Pharmacopoeia. Pharm. Pharmcoel, Lett., 9(2): 48-51.

British Pharmacopoeia, (1963). Determination of volatile oil in drugs. The Pharmaceutical press,17 Bloomsbury square. Lond. WCI.anaerobic treatment of source-separated household waste as fertilizer Diacono, M. and F., Montemurro. , Long-term effects of organic amendments on soil fertility, In : Sustainable Agriculture, Volume 2(Ed):E. Springer Netherlands, pp. 761-786. 
Ewees, M.A., A.sh. Osman and D.M. El Sowfy,(2008) significance of applied organic manure combined with N- mineral fertilizer. Egypt.J.Soil Sci.48, No.3 pp.347-360

Fathy, M. S., A. M. Shehata, A. E. Kaleei and S. M. Ezzhat (2002). An acylated Kaempferai glycoside from flavenes of Foeniculum vulgare and F. daice. Moecules, 7: 245- 251.

Gomez, K. A. and A. A. Gomez (1984). Statistical Produces for Agricultural Research 2nd Ed. John Wiley \& Sons Inc. New York.

Haraldsen, TK., U. Andersen, T. Krogstad and R. Srheim,(2011), Liquid digestate from to barely . Waste Monag. Res.29(12):12711276.

Jensen, ME.(1983). Dessign and operation of farm irrigation systems`An ASAE Monograph No. 3.

Kandeel, Y. R., R. S. Nofal, F. A. Menesi, K. Reda; M. Taher and Z. T. Zaki (2001). Effect of some cultural practices on group and chemical composition of Foeniculum valgare Mill. Proc. 5th Arab. Hort. Conf. Ismailia, Egypt, March 24- 28 pp. 61- 72.c

Massoud, H. Y. A. (2007). Evalution of some varieties of Ocimum basilicum, L. and Their response to some organic manures. J. Agric. Sci., Mansoura Univ., 32 94). 2863- 2880.

Maunksela, L., M. Herranen and M. Troniainen, 2012. Quality assessment of biogas plant and products by plant bioassays. Int J. Environ. Sci Devlop., 3(3)305-310.

Michael, A. M. (1978). Irrigation theory and practice. Vikas phb. House PVTLTD New Delhi, Bombay: 360 p. 
Mohamed, S. A.(2006), A compression between the effect of different source of organic and mineral fertilizes on growu, anatomical structure yield and chemical composition of Roselle $(\mathrm{H}$. biscus sabdriffall) plant. The second conference on farm Integrated pest Management. Jan, 16-18,2006, Fayoum. Egypt.

Page, A. L; R. H. Miller and D. R. Keeney (1982). Metholds of Soil Analysis. chemical and microbiological properties. Part 2 2nd Ed. ASA- SSSA. Madison, Wisc, USA.

Pokurna, K. J. (1984). Effect of long term fertilization on the dynamics of changes of soil organic matter zenter- ablatt fir Mikrobiolkogie. 139 (7): 497- 504 (Soil and Fertile. Abst., 45, 3225).

Sadej W., Przekwas K. (2008): Fluctuations of nitro- gen levels in soil profile under conditions of a long- term fertilization experiment. Plant Soil Environ.,. 54: 197-203.

Sarker, M. C., m., singh, and J. Nath, (1992). Influence of foray and manure on soil breccoi and cauliflower to water and nitrogen in the desert herl. Science 31(2):201-205.

Weier, K.L., Doran, J.W., Power, J.F. \& Walters, D.T. (1993). Denitrification and the dinitrogen/nitrous oxide ratio as affected by soil water, available carbon, and nitrate. Soil Science Society of America Journal 57(1), 66-72.

Wichtl, M. and N. G. Bisset (1994). Herbai druge and phytopharmaceuticeles (ed.) Med. Pharm Scientific Pubi Stuttgart pp. 107- 108.

Zhong, W., T Gu, W. Wang, B. Zhang, X. Lin, Q. Hang and W.Shen. 2010. The effects of mineral fertilizer and organic manure on soil microbial community and diversity. Plant soil , 326(1) :511-522. 
وزارة الزراعة و استصلاح الأر اضى ـ مركز بحوث الصحر اءه ـ ـ إدارة التدريب إعداد د باسر

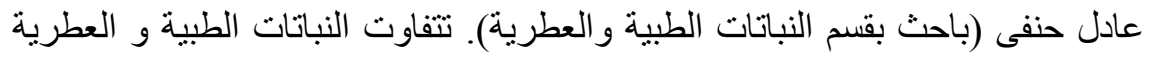

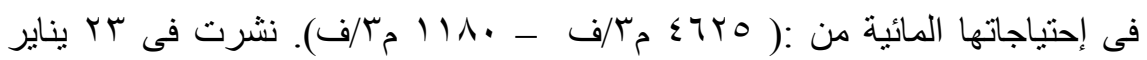

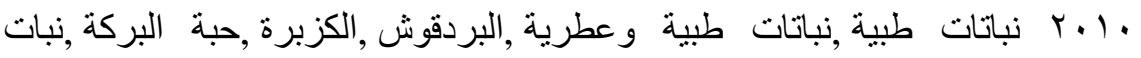

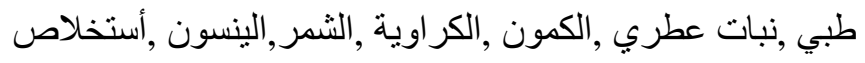

\section{الملخص العربى}

\section{سماد البيوجاز السائل وتأثيره على انتاجيه محصول الشمر ونسبه الزيت}

\section{المستخرج تحث مستويات مختلفة من مياه الرئ الري}

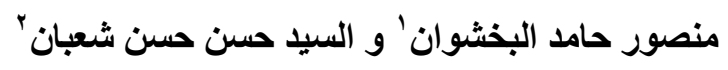

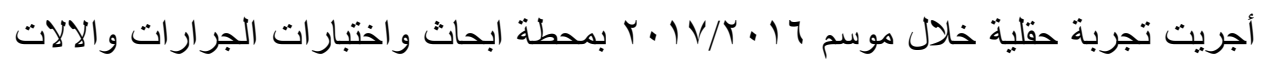

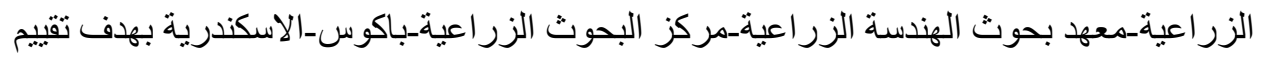

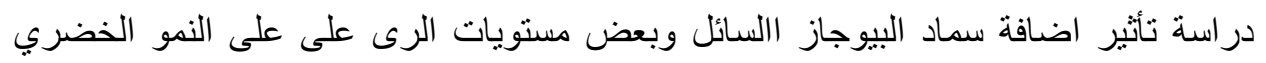
و التكوين الكيميائي والزيوت الأساسية لمحصول الثنمر. حيث يسمى الخليط التبقى من عمليه

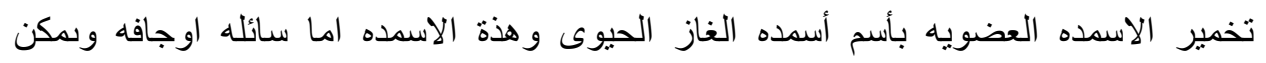

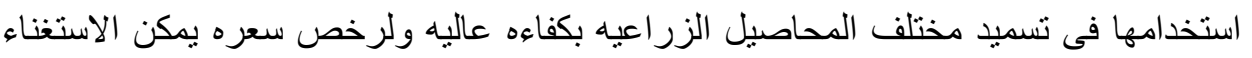

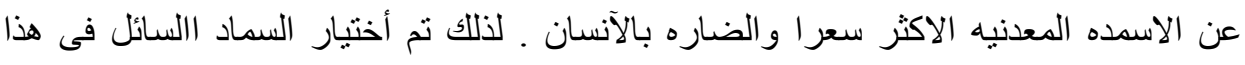

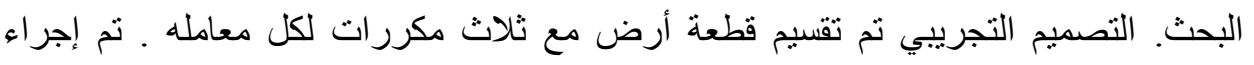

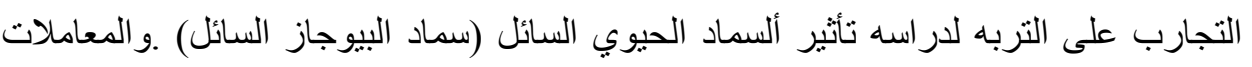

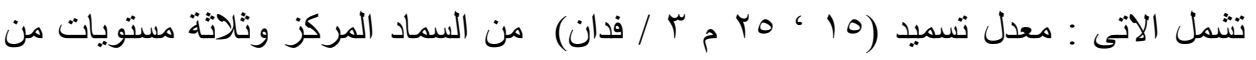

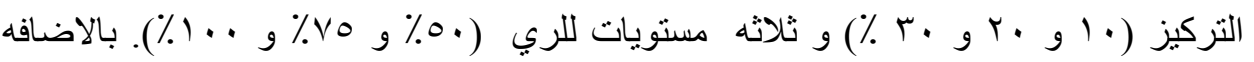

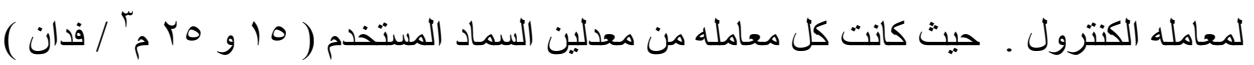

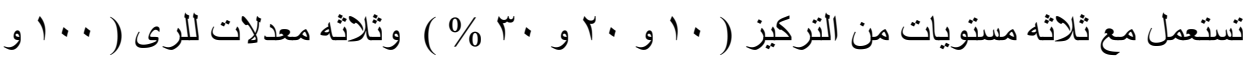

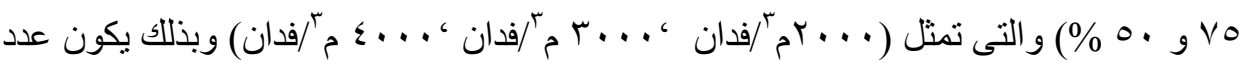

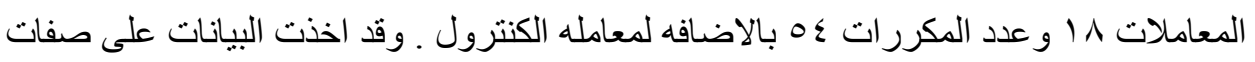

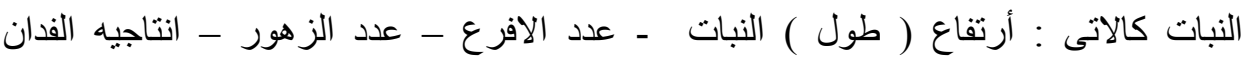

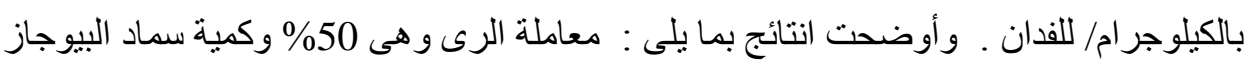

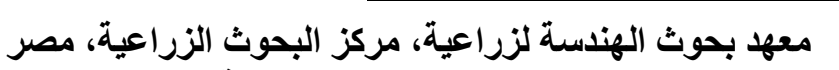

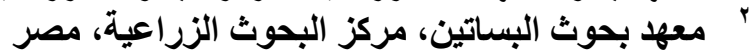




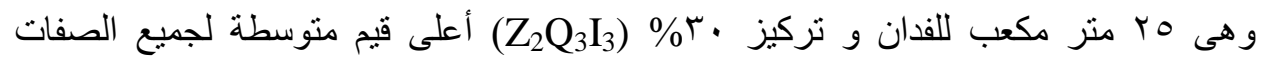

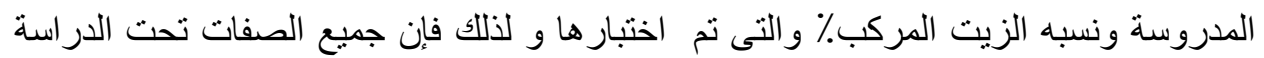

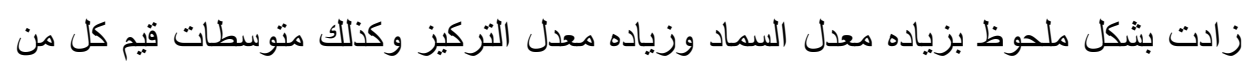

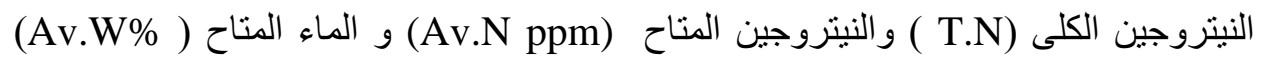

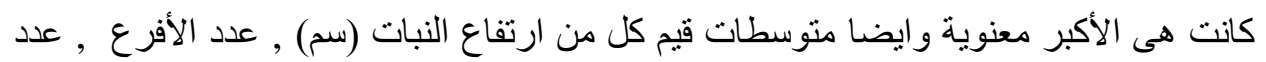

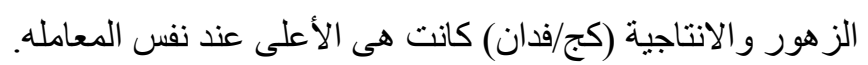

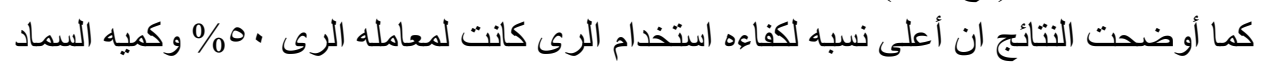

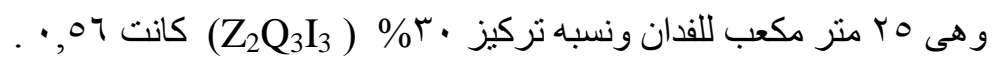

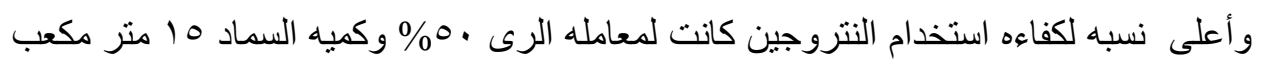

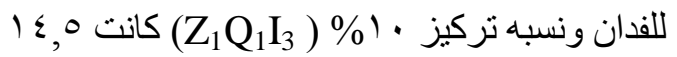

